\title{
Tremor Compensation by Use of a Mechatronic Cup Holder
}

\author{
M. Fischer, M. Schraufstetter, C. Richter, F. Irlinger, T. C. Lueth \\ Dept. Micro Technology and Medical Device Technology \\ Technische Universität München \\ Munich, Germany \\ Marcus.Fischer@tum.de
}

\begin{abstract}
In this paper a new assistive device for persons suffering tremor disease is presented. It should help those people to get along with the everyday life situation of consuming beverages and replace the feeding cups currently used.

The cup holder is built up as a mechatronic system. It consists of a kinematic structure sustaining a standard cup as well as electronic and software components. The cup is moved by the device in order to compensate linear and rotational accelerations as well as tilt induced by a person's tremor. For this purpose the characteristics of the fast and large scaled tremor movements of the patient's hand are measured first. By use of the inverse kinematics calculated for the system and a method for the modelling of the liquid's movement, necessary reactions of the cup holder are deduced. Using this information the device's motors are actuated to avoid spillage of the liquid. The underlying calculations are run on a micro controller integrated in the system.
\end{abstract}

An experiment was conducted to validate the function of the system. For this purpose an industrial robot was used to apply common tremor frequencies and amplitudes to the cup holder. It was shown that by use of the compensatory cup holder the spillage of the beverage can be eliminated at a wide range of different tremors.

Keywords- tremor disease; compensation; assistive device; cup holder; mechatronic;

\section{INTRODUCTION}

Tremor is a rhythmic, oscillating movement of a part of the human body referring to a middle position [1]. It has many different reasons and appearances. Beneath the physiological tremor there are some other types which for example result from diseases or pharmaceuticals [2]. Most common are the Parkinson and the essential tremor [3].

The different types of tremor vary regarding to the part of the body concerned, the frequency, and intensity. The physiological tremor appears at low amplitude of vibration and at high frequency. Amplitudes of tremors resulting from diseases are higher. Tremor resulting from the Parkinson disease is a typical resting tremor. It occurs at a frequency of $3-10 \mathrm{~Hz}$. By contrast the essential tremor causes oscillations at $4-12 \mathrm{~Hz}$ and occurs when the affected muscle is in action. It can especially be seen whilst grasping something using the hands [3]. Beyond, there are the tremors occurring during intended movements and the cerebellar tremor which appear when for example heading the hand towards a defined goal position [2].

Permission to make digital or hard copies of all or part of this work for personal or classroom use is granted without fee provided that copies are not made or distributed for profit or commercial advantage and that copies bear this notice and the full citation on the first page. To copy otherwise, to republish, to post on servers or to redistribute to lists, requires prior specific permission and/or a fee. PERVASIVEHEALTH 2010, March 22-25, Munchen, Germany

Copyright (C) 2010 ICST 978-963-9799-89-9

DOI 10.4108/ICST.PERVASIVEHEALTH2010.8885
Because of the huge number of different causes of tremor diseases, their classification and medication is a demanding task. A specific treatment and healing is hard to achieve. That is why people suffering from tremor disease are critically handicapped in everyday life. Especially ingestion is affected by the unintentional movements. The consequences are highly perceptible when a beverage is spilled while drinking.

\section{STATE OF THE ART}

In practice people suffering tremor disease can use feeding cups for drinking. That way, the problem of spilling beverages is reduced as far as possible, due to the narrow mouthpiece. But this particular characteristic of the feeding cups causes different problems. High effort has to be taken to guide the small mouthpiece to the mouth. Beyond, sensory impressions like the temperature of the beverage or its smell are reduced. Additionally the use of feeding cups holds a danger of aspiration of the beverage [4].

Hence solutions have to be developed to support people in these situations, e.g. by balancing the beverage and therewith the reduction of sloshing caused by motion.

In general, there are several possible solutions for this task that can be classified into mechanic and mechatronic ones. Damped spring-mass systems and pendulums for example are types of mechanic systems. In mechatronic systems which are used for this purpose the spring and damper are replaced by active actuators.

Examples for passive damped spring-mass systems can be found in [5] and [6]. Both systems try to keep a specific element in its rest position. The first example describes a seismographic system in general with its seismic mass, the spring characteristic and the damping function. The second application is the so called steadicam ${ }^{\circledR}$, a stand for film cameras. It balances movements caused by human influence like tremor and walking. It uses the moment of inertia and gravity as restoring forces.

Furthermore, there are examples for active systems which balance liquids. In those systems actuators are used to realize the balancing movements. To control them, the current states of both, the system and the liquid, have to be known. In [7] an example for the modelling of fluid motion has been proposed in order to examine different shapes of fuel tanks. The oscillations caused by external forces have been modelled as first order oscillations. 
An application in the field of robotics can be found in [8]. For the automation of a transport system, occurring 2dimensional motion of liquid steel in containers has been modelled to realize a so called sloshing control. In $[9,10]$ this area of research has been continued with the adoption to different levels of the fluid and a second degree of freedom (DOF) of the transport system. The fluid's movement was represented by a pendulum model. Another active method for the compensation of accelerations was presented in $[11,12]$. Accelerations which affect a load placed on a mobile platform were actively compensated by use of a parallel kinematics built underneath. The platform was, for short impulses, able to induce accelerations opposite to the moving direction. For horizontal forces which have a longer lasting influence, the platform could tilt using gravity to cause acceleration. In [13] online motion planning is proposed for a 1-DOF kinematics carrying fluids. In [14] signals of acceleration and gyro sensors have been used as an input for the compensation of accelerations. An augmented pendulum algorithm containing a damping function was used to imitate the movement of a passive pendulum by means of an actuated platform.

As it can be seen in those examples, in most cases mechanic solutions are the cheapest and easiest way for the compensation of accelerations. But those systems can hardly be adapted to different situations as for example different levels of beverages in a cup. By contrast mechatronic systems, especially the ones using online path planning, can be adapted to different situations. For this purpose occurring movements have to be detected. Relating to the tremor compensation the tremor's amplitude and frequency have to be determined.

For this task different methods are known. Mechanical devices like the Edward-Sphygmograph [15] or an instrument which uses potentiometers to define the position of a person's finger [16] have been developed to record the movements. The latter uses a spatial mechanism and its inverse kinematics to define the finger's position. Recent systems use techniques such as electromyography, accelerometry, laser vibrometry, or camera and ultrasonic systems. Electromyography for example is used in [3] to measure the rhythm and frequency of the tremor of a human's fore arm. The determination of the tremor's amplitude is rather imprecise using this method. Another type of tremor measurement, the videometry, uses high speed video or infrared cameras to observe the movement of predefined markers on the analysed part of the body like for example shown in [17]. Non-contact but expensive methods use ultrasound or laser vibrometry as for example proposed in [18]. By use of the ultrasonic methods a real time recording of the tremor can be carried out. All of those measurement methods require specific equipment and fixtures. Hence, the measuring of the tremors' characteristics can only be conducted in laboratories. One ambulant method, as it is necessary for the assistive device considered in this paper, is accelerometry. It can be found in systems like Digi Trac ${ }^{\circledR}$, which is analysed in [19]. The system is attached to the analysed part of the body by use of rubber bands or Velcro®. It allows for the monitoring of a 3-dimensional tremor movement. To ensure portability, the system's energy supply is realised by use of accumulators. The identified movement data is recorded onto internal storage and afterwards evaluated using a computer. The same sensors can also be used to measure the current orientation of parts of the body as shown in [20]. For this purpose the sensors were affixed to human legs. Joint accelerations could be determined.

In clinical applications tremor compensating methods are for example used in retina surgery. For that matter the tremor of the surgeon's hand is compensated by hand held surgical instruments [21-23]. The current task of the tremor compensating cup holder differs significantly from these high precision applications. It comprises large scaled ranges of movement.

\section{MATERIAL AND METHODS}

The objective of the current work is the development of a mechatronic cup holder that can be used to compensate the effects of the hand's tremor during drinking situations. Therefore, a cup is fitted to an actuated mechanism. The movements of the beverage contained in the cup, which are evoked by the tremor, should be balanced by the motion of the device's kinematics. This way spillage of the fluid should be avoided. To reduce the effects of tremor, the cup holder detects and compensates occurring accelerations and inclines. It can be used at high frequencies, fast accelerations and in comparison to state of the art medical systems at particularly large amplitudes caused by a disease's tremor. Acceleration sensors are used to gather information on the current position and orientation of the device as well as occurring accelerations.

\section{A. Kinematics design and inverse kinematics}

The kinematic structure of the device is designed as a parallel robot. It deploys two degrees of freedom. Two servo motors are used to actuate the system and accelerate the fluid opposite to the motion induced by the tremor.

In Fig. 1 the cup holder's kinematic structure is shown. The ring 6 is used to hold a cup. It is supported by one single ball joint integrated in the non-moving framework and a two arm parallel mechanism. The two arms include one revolute and one ball joint (the kinematics' arm containing link 2 and 3) or two ball joints respectively (the arm containing link 4 and 5). The total DOF of the system is three. With each of the actuators only one degree of freedom can be served. The residuary one is an axial rotation occurring around the center axis of link number 5. Since the impact of this rotation is very small it is neglected in the following.

To compensate the liquid's sloshing, as described later on, the orientation of the cup is changed. For this purpose the position and orientation of the ring 6 is changed by use of the actuators. To calculate the necessary angularities of the actuators the inverse kinematics of the system has to be identified. Its definition necessitates the introduction of several coordinate systems (Fig. 2).

The first system (sys) is fixed to the framework of the device. It remains unmoved relating to the body. For zero position of the actuators the center of the ring equals the center of this coordinate system. Its $x / y$-plane is oriented horizontally. 


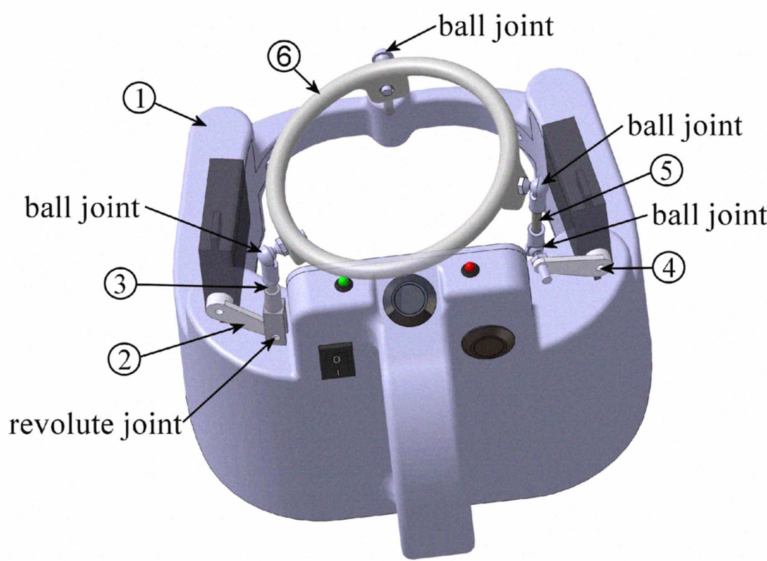

Figure 1. Model of the system's kinematic structure showing its framework (1), the single transmission elements (2-6) and its joints.

Each of the remaining transmission elements comprises two coordinate systems. One system $\left(A_{i}\right)$ marks the reference position of element $i$ relative to the foregoing element. The second one $\left(B_{i}\right)$ identifies the element's actual orientation. The basic system $A_{i+1}$ of element $i+1$ is defined with respect to $B_{i}$. The movements occurring in the joints or the necessary translations caused by the geometries of the single elements are represented as homogeneous transformation matrices. A matrix ${ }^{A} \mathbf{T}_{B}$ for example characterizes a transformation from coordinate system $A$ to $B$, with

$$
{ }^{A} \mathbf{T}_{B}=\left(\begin{array}{cc}
{ }^{A} \mathbf{R}_{B} & { }^{A} \mathbf{t}_{B} \\
\mathbf{0}^{\mathrm{T}} & 1
\end{array}\right)
$$

The calculation of the angularity of the two servo motors of both actuated arms of the cup holder was considered simplified as a 2-dimensional geometric problem. For this purpose, the ball joint between links 4 and 5 (Fig. 1) has been substituted by a revolute joint in the calculation. This is possible, since the system's movement into the direction of the third DOF is rather small and therefore can be neglected. To be able to realize a time efficient calculation the inverse kinematic problem was further reduced to a symmetric one. Hence in subsequent calculations both arms of the robot are regarded equal. This way the number of calculations can be reduced significantly. This reduction is especially necessary to minimize the calculation effort of the micro controller.

For setting up the inverse kinematics, the rotations from coordinate system $A_{6}$ to $B_{6}$ describing the rotation of the cup holder's ring has to be formulated. Afterwards the position of the point $\mathbf{p}_{1}$ has to be expressed regarding to the angularity $\alpha$ of the motor, the link lengths, and the overall geometry of the cup holder. With respect to the geometric dimensions and the aforementioned simplifications its position can be defined as

$$
{ }^{A_{2}} \mathbf{T}_{s y s} \cdot{ }^{s y s} \mathbf{T}_{A_{6}} \cdot{ }^{A_{6}} \mathbf{T}_{B_{6}} \cdot{ }^{B_{6}} \mathbf{p}_{1}={ }^{A_{2}} \mathbf{p}_{1}:=\left(\begin{array}{c}
p_{1_{x}} \\
p_{1_{y}} \\
0
\end{array}\right) .
$$

The transformations ${ }^{A 2} \mathrm{~T}_{s y s},{ }^{\text {sys }} \mathrm{T}_{A 6}$ and ${ }^{B 6} \mathrm{~T}_{p l}$ are known by consideration of the links' dimensions. ${ }^{A 6} \mathbf{T}_{B 6}$ and ${ }^{A 2} \mathbf{p}_{l}$ have to be determined. Because of the geometric symmetry of the cup holder the postulated transformation ${ }^{A 6} \mathbf{T}_{B \sigma}$ around the ball joint between the framework 1 and the ring 6 is restricted to rotations around the $\mathrm{x}$ - and $\mathrm{y}$-axis of coordinate system $A_{6}$. With the two angles of rotation called $\beta$ and $\gamma$ respectively, the transformation is:

$$
{ }^{A} T_{6} T_{B_{6}}=\left(\begin{array}{cccc}
1 & 0 & 0 & 0 \\
0 & \cos \gamma & -\sin \gamma & 0 \\
0 & \sin \gamma & \cos \gamma & 0 \\
0 & 0 & 0 & 1
\end{array}\right) \cdot\left(\begin{array}{cccc}
\cos \beta & 0 & \sin \beta & 0 \\
0 & 1 & 0 & 0 \\
-\sin \beta & 0 & \cos \beta & 0 \\
0 & 0 & 0 & 1
\end{array}\right) .
$$

By use of the vector ${ }^{A 2} \mathbf{p}_{l}$ and the length of the kinematics' elements the angularity of actuator 1 can be calculated. First, the law of cosines is used to calculate $\varphi_{1}$ :

$$
\varphi_{1}=\operatorname{acos} \frac{\left|{ }^{B_{2}} \mathbf{t}_{A_{3}}-{ }^{A_{2}} \mathbf{p}_{1}\right|^{2}-\left|{ }^{s y s} \mathbf{t}_{A_{2}}-{ }^{B_{2}} \mathbf{t}_{A_{3}}\right|^{2}-\left|{ }^{s y s} \mathbf{t}_{A_{2}}-{ }^{A_{2}} \mathbf{p}_{1}\right|^{2}}{-2 \cdot\left|{ }^{s y s} \mathbf{t}_{A_{2}}-{ }^{B_{2}} \mathbf{t}_{A_{3}}\right| \cdot\left|{ }^{s y s} \mathbf{t}_{A_{2}}-{ }^{A_{2}} \mathbf{p}_{1}\right|}
$$

In the following the substitute angle $\omega_{l}$ is geometrically defined as shown in Fig. 2. It can be calculated using

$$
\omega_{1}=\operatorname{acos}\left(\frac{\left(\begin{array}{lll}
0 & 1 & 0
\end{array}\right)^{\mathrm{T}}{ }^{{ }^{A}}{ }_{2} \mathbf{p}_{1}}{\left|\left(\begin{array}{lll}
0 & 1 & 0
\end{array}\right)^{\mathrm{T}}\right| \cdot{ }^{A_{2}} \mathbf{p}_{1}}\right) .
$$

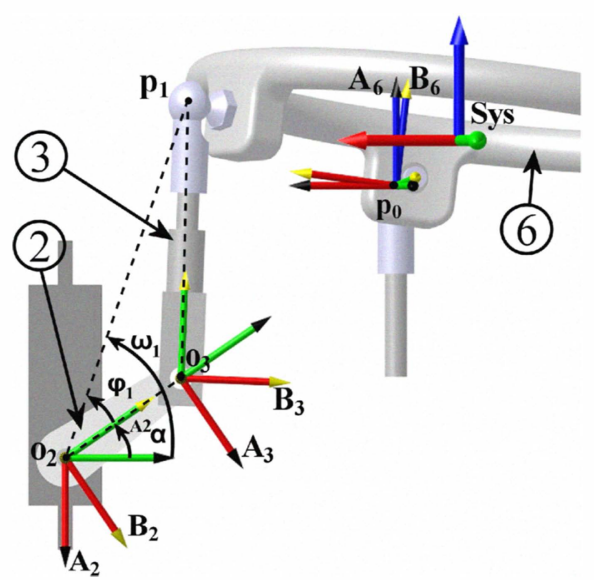

Figure 2. Representation of the geometric correlation of the motor's angularity $\alpha$ and the movement of the ring (6) holding the cup. Additionally

the elements 2 and 3 of one arm of the kinematics as well as their reference $(A)$ and actual $(B)$ coordinate systems are shown. 
By use of these two angles the resulting angularity $\alpha$ for motor 1 can be calculated using

$$
\alpha=\omega_{1}-\varphi_{1}
$$

The calculation of the position of point $\mathbf{p}_{2}$ on the opposite side of the ring is done analogously, since the three degrees of freedom of the lower ball joint of link number 5 are reduced by one. After the mathematic definition of the two arms of the cup holder, its inverse kinematics can be calculated with respect to the two angularities of the servo motors.

\section{B. Determination of the cup holder's accelerations}

To compensate the accelerations that act upon the beverage, those who affect the cup holder have to be determined. For this purpose, four acceleration sensors (Bosch SMB 380®) serving three orthogonal directions each were integrated into the device's framework (Fig. 3). By use of the twelve sensor signals obtained and a calculation rule proposed in [24] not only the relative position, velocity and the linear but also the angular acceleration of the cup holder can be calculated. The latter are needed for the determination of the system's orientation by means of the Kalman filter, which is described in paragraph D.

Without consideration of the derivations of the position vector $\mathbf{r}$ with respect to time the acceleration of every point $\mathbf{p}$ of the non-moving framework of the cup holder can be calculated to:

$$
\mathbf{a}=\mathbf{a}_{0}(t)+\dot{\omega} \times \mathbf{r}+\omega \times(\omega \times \mathbf{r})
$$

Therein a represents the vector of acceleration for a definite point of the system, $\mathbf{a}_{0}(t)$ the acceleration of the origin's coordinate system, $\boldsymbol{\omega}$ the angular rate vector of the body, and $\mathbf{r}$ the geometric coherence between the single point and the origin. The dot product of a and the vectors of the effective directions $\mathbf{e}_{i}$ of the sensor axes (8) results in a system of 12 equations. It solely contains known components like the geometric position $\mathbf{r}$ and the orientation of the sensors' 12 principal axes $\mathbf{e}_{i}$ :

$a_{i}=\mathbf{a} \circ \mathbf{e}_{i}$.

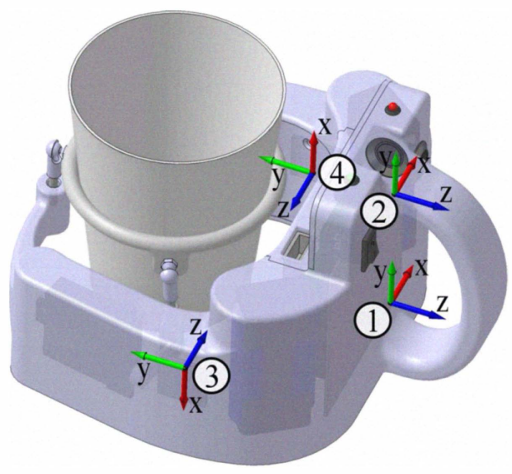

Figure 3. Positioning of the four acceleration sensors with three sensitive axes each. The sensors must not be positioned within a single sectional plane, the axes have to be aligned parallel or orthogonally.
Since the system's overall dimensions are small, the distances in between the sensors are small, too. Hence, the sensor measurements have to be sufficiently accurate. Therefore a calibration of the sensors is very important to compensate inaccuracies due to the assembly process. During this calibration the cup holder was put into three definite orientations $\left(0^{\circ}\right.$, $+45^{\circ}$, and $-45^{\circ}$ ) relative to the world coordinate system (cf. Fig. 6). The sensors' signals resulting from gravity were determined (Fig. 4). Since the data which was measured by means of the sensors did not correspond to the expected values, the parameters for offset $o$ and gain $k$ had to be defined for every sensor and every axial direction $(x, y$ and $z$ ). For each arrangement $j$ the following mathematic correlation was set up:

$$
\left(\begin{array}{l}
a_{\text {theo }, x_{j}} \\
a_{\text {theo }, y_{j}} \\
a_{\text {theo }, z_{j}}
\end{array}\right)={ }^{s y s} \mathbf{R}_{j} \cdot\left(\begin{array}{l}
k_{x_{j}} \cdot a_{m, x_{j}}+o_{x_{j}} \\
k_{y_{j}} \cdot a_{m, y_{j}}+o_{y_{j}} \\
k_{z_{j}} \cdot a_{m, z_{j}}+o_{z_{j}}
\end{array}\right) .
$$

Within the equation, $a_{\text {theo }}$ refers to the expected acceleration in every of the three axial directions (sys coordinate system), $a_{m}$ to the accelerations measured with the sensors for each direction and ${ }^{s y s} \mathbf{R}_{j}$ to the rotation matrices of the sensors' coordinate systems with respect to the sys system.

Since it was not possible to solve the system out of nine unknown variables analytically, the Newton-Algorithm was used to define the variables.

\section{Sensor signals gained in different situations}

In a first test the designed system was validated. The sensors' signals in different states of the system were recorded and assigned to different use cases. For this purpose test persons had to use the cup holder in a scenario of everyday life. First they had to pick up the device, thereupon walk around at different velocities and then drink using the system. At last the test persons had to deposit the device on a table.

During the time the cup holder was still on the table, the measured magnitudes of acceleration of every single axis $(x, y$ and $z$ ) only showed the noise of the sensor signals. When the system was lifted up the biggest impact could be seen on the $z$ axis' signal. The other axes only showed a minor reaction dependent on how straight the upward movement was realized. The following phase of walking could be seen as peaks of acceleration in each of the sensors' signals, primarily in $z$ direction (Fig. 5).

With the sensor data gathered in this analysis a Fourier transform was carried out. Hereby, frequencies recorded during the tests could be sorted regarding to their frequency of occurrence at different steps of the scenario. The noise of the sensors could be found in different frequency ranges, mainly between 0 and $15 \mathrm{~Hz}$. 

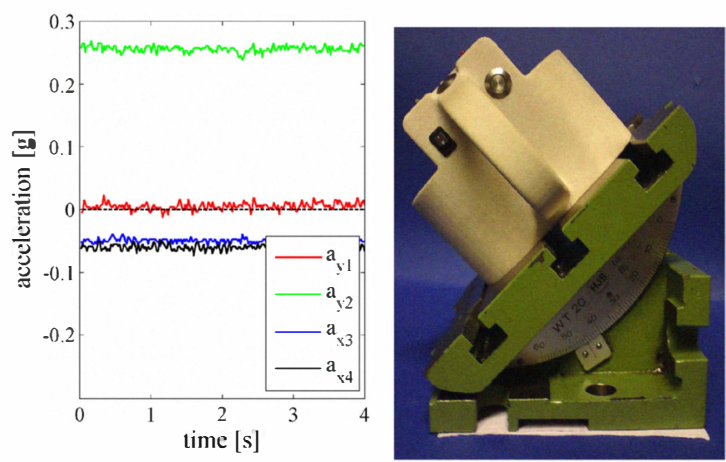

Figure 4. The four sensors' acceleration signals towards the $y$-axis of the sys coordinate system (horizontal arrangement of the cup holder) before the calibration (left); positioning of the cup holder at $45^{\circ}$ for the calibration (right).

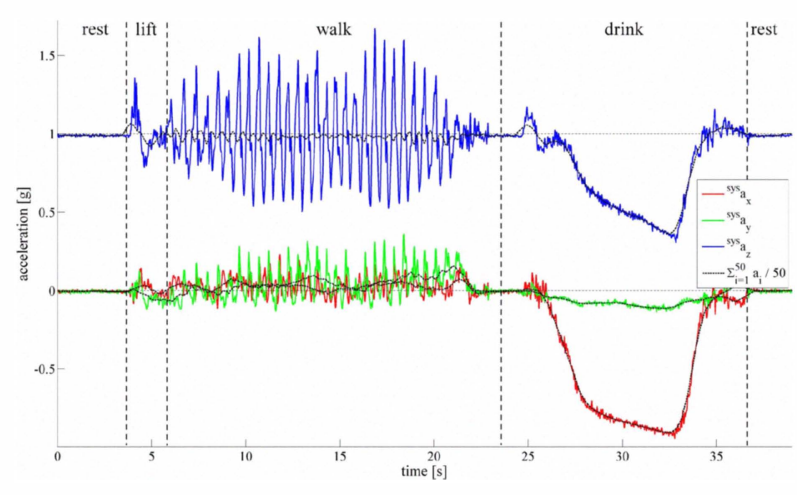

Figure 5. Sensor data for the acceleration in $x, y$ and $z$-direction: noise can be seen when the cup holder is at rest; an impact, primarly on the signal in $z$-direction (upper signal), occurs during the lift of the system; peaks resulting from the steps of the test persons can be seen during the walking phase; whilst drinking, the middle value differs from zero, accelerations change constantly.

A distinct peak in the Fourier transform could be found at about $2 \mathrm{~Hz}$ during the walking phase. This complies with the frequency of the test persons' steps. In test situations when the test persons walked at higher velocity, the Fourier transform showed a smaller peak at higher frequency. Tremor can mostly be found in the range from 3 to $12 \mathrm{~Hz}$. Since there was no intentionally conducted tremor only small peaks of physiological tremor could be detected. Those were approximately the same in all three directions of space.

The moving average of the accelerations stayed around zero during most of the test scenarios. Merely in the last step of the test, where the test persons drank and intentionally tilted the device, it changed significantly. It declined for the $z$-direction and inclined for the other two directions. The sense the cup holder is turned can be determined by evaluation of the alignment of the gravity vector.

\section{Calculation of the system's orientation}

The cup holder's orientation can be described as a rotation of the sys-coordinate system fixed to its framework (Fig. 2) around the world coordinate system. For the calculation of the orientation, the information about the system's accelerations gathered by means of the acceleration sensors (paragraph B) is used. Considered statically, the elements of the acceleration vector indicate the direction of the vector of gravity $g$. The twist of the sys coordinate system compared to the world system can be calculated. To ensure consideration of the correct quadrant, the atan 2 function has been used. The angles $\gamma$ and $\beta$ around the world $x$ - and $y$-axis have been calculated by use of (10) and (11).

$$
\begin{aligned}
& \text { world }=\operatorname{atan} 2\left({ }^{\text {sys }} g_{y},{ }^{\text {sys }} g_{z}\right) . \\
& { }^{\text {world }} \beta=-\operatorname{atan} 2\left({ }^{s y s} g_{x},{ }^{s y s} g_{z}\right) .
\end{aligned}
$$

Considered dynamically, in addition to gravity, the acceleration of the tremor and the usual movements affect the measured data. These interfere with the accelerations caused by gravity. So an additional method for filtering the data and reducing this sensor noise had to be implemented. Hence, a Kalman filter has been used. During the time update phase the angles around the $x$ - and $y$-axis as well as their derivations are estimated using a system model and the angular accelerations (section III, B) as input information. In the measurement update phase the angles of rotation $(10,11)$ are determined by means of the sensors. The identified values differ from the real values due to noise occurring during the measurement. The different errors which have to be considered originate from the noise of sensors as well as the discretization.

\section{E. Simulation of the fluid's movement}

The different orientations as well as accelerations which arise during the use of the device lead to different angles between the surface of the liquid and the horizontal plane of the world coordinate system. This can eventually lead to spillage of the beverage. When the effect of the acceleration stops, the liquid swings back into the opposite direction. A damped oscillation succeeds.

If the oscillation of the liquid should be compensated, its surface inclination has to be known. This could be achieved by integrating sensors in the cup which would be difficult to arrange due to hygienic problems and high cost. Additionally the washability would have to be assured.

A more practicable solution is the modelling of the liquid's movement. For that purpose a pendulum model can be used to describe the liquid's level (Fig. 6).

The model used for the cup holder is based on methods shown in [10]. The heights of the liquid $l_{1}$ and $l_{2}$ have to be levelled $\left(l_{l}=l_{2}\right)$ by a correlating movement of the cup holder. The equation of the liquid's model, e.g. for the $x$-direction of the sys coordinate system was compiled to

$$
\ddot{\theta}_{x}=-\frac{c}{m} \cdot\left(\dot{\theta}_{x}-\dot{\eta}_{x}\right)-\frac{g}{l} \cdot \theta_{x}-\frac{1}{l} \cdot a_{x}
$$




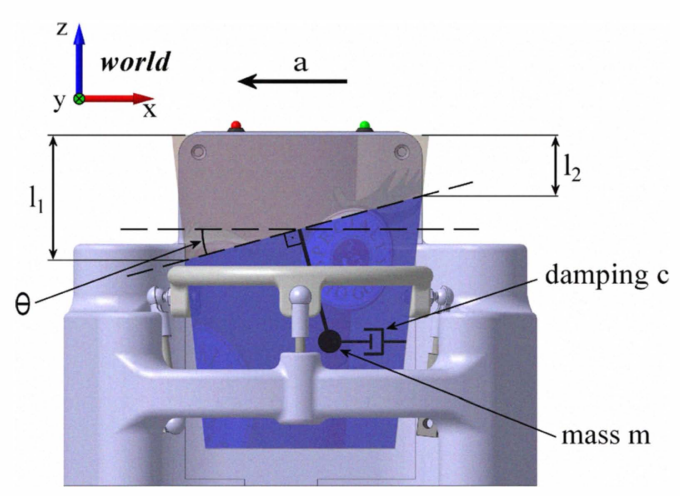

Figure 6. Calculation of the beverage's movement by use of a pendulum model with the heights $l_{1}$ and $l_{2}$ as well as the angle $\theta$, resulting from an acceleration $a$ on the pendulum with its mass $m$ and the damping $c$.

The model accounts for the acceleration $a_{x}$, the gravity $g$, the angular rate $\dot{\eta}_{x}$ of the ring of the cup holder (respecting a down time for the actuator movement), the mass $m$ of the fluid and the angle of the fluid $\theta$ which results from the heights $l_{I}$ and $l_{2}$. The length of the pendulum $l$ results from the eigenfrequency of the fluid. The coefficient $c$ takes into account the viscosity of the fluid and the friction between fluid and cup. It is derived experimentally. The equation has been used for the $y$-direction analogously. For the following calculations the accelerations, which are measured in the world coordinate system have to be transformed into the current system sys by use of the transformation ${ }^{\text {world }} \mathbf{T}_{\text {sys }}$ (13).

$$
{ }_{\text {world }} \mathbf{T}_{\text {sys }}=\left(\begin{array}{cccc}
1 & 0 & 0 & 0 \\
0 & \cos \gamma & -\sin \gamma & 0 \\
0 & \sin \gamma & \cos \gamma & 0 \\
0 & 0 & 0 & 1
\end{array}\right) \cdot\left(\begin{array}{cccc}
\cos \beta & 0 & \sin \beta & 0 \\
0 & 1 & 0 & 0 \\
-\sin \beta & 0 & \cos \beta & 0 \\
0 & 0 & 0 & 1
\end{array}\right)
$$

Additionally the constants of (12) have to be defined. The gravity index is specified to $9.81 \mathrm{~m} / \mathrm{s}^{2}$. Since the mass of the cup varies with the level of the liquid (which changes during the use of the cup holder) a default value was defined to $0.2 \mathrm{~kg}$. It represents the weight of the maximum possible fluid level which represents the situation of maximum danger of spillage. The coefficient $c$ was defined experimentally to $0.54 \mathrm{Ns} / \mathrm{kg}$ according to the procedure presented in [10]. The eigenfrequency of the first mode of the liquid was calculated to $1.64 \mathrm{~Hz}$. Referring to the procedure the length of the pendulum was assumed to be $0.092 \mathrm{~m}$.

\section{F. About the mechatronic and mechanic realization}

For the validation of the mathematical models and the functionalities of the tremor compensating cup holder concept a fully working prototype was built up. For its fabrication rapid prototyping as well as standard manufacturing methods have been used. The base frame, in which the sensors have already been built in, has been fabricated by means of 3D-printing. For the additional kinematic elements like joints or links, standard parts have been used. The actuation has been realized using digital servo motors running at $6 \mathrm{~V}$ with a maximum torque of $0.35 \mathrm{Nm}$. The motors have an integrated position controller which is fed by a PWM-signal. Since the cup holder should be a mobile system, the power supply, the control unit, and all electronic peripherals had to be integrated into the system. The controller was implemented on an 8 bit micro controller (ATMega 2560®).

The micro controller $(16 \mathrm{MHz})$ calculates the current angle $\theta$ of the liquid's surface in $x$ - and $y$-direction (12), the cup holder's current orientation as well as the inverse kinematics (6) of the system. Knowing the state of the system, the necessary movement of the cup can be deduced. It is moved such that the liquid's surface is ever perpendicular to the cup's center line. Using the inverse kinematics the necessary angles of rotation of the two servo motors to obtain this orientation are calculated.

For the implementation on the micro controller the mathematical problem in (12) had to be discretized. Additionally it has to be formulated as a 2-dimensional system of first order equations according to

$$
\left(\begin{array}{l}
\dot{z}_{1} \\
\dot{z}_{2}
\end{array}\right)=\left(\begin{array}{cc}
-\frac{c}{m} & -\frac{g}{l} \\
1 & 0
\end{array}\right) \cdot\left(\begin{array}{c}
z_{1} \\
z_{2}
\end{array}\right)+\left(\begin{array}{c}
\frac{c}{m} \cdot \dot{\eta}+\frac{1}{l} \cdot \text { world }_{a} \\
0
\end{array}\right) \text {, }
$$

with $z_{1}:=\dot{\theta}$ and $z_{2}:=\theta$. For the numerical solution of this equation system the two step procedure was used. Its rule is shown in (15) with the function $f$, the discretized steps $k$ and $k+l$ as well as $h$ as the step size between the steps $t_{k+l}$ and $t_{k}$.

$$
z_{k+1}=z_{k}+\frac{h}{2} \cdot\left(3 \cdot \mathrm{f}\left(t_{k}, z_{k}\right)-\mathrm{f}\left(t_{k-1}, z_{k-1}\right)\right)
$$

\section{EXPERIMENTS}

To test the cup holder's operability, a number of experiments has been conducted using the prototype. One precondition for the tests was the provision of identical, reproducible input parameters. Additionally, the movement's parameters should be equal to those occurring under real conditions. During the first part of the experiments the device was moved with the orientation and acceleration compensation switched off. Subsequently, the prototype was moved with the compensation function switched on. The behaviour of the liquid was monitored in both situations.

Since reproducibility was required for these first experiments no people were involved. Instead an industrial robot, a Stäubli RX 90, was used to simulate a possible tremor movement. This implied that the robot could generate the dynamic properties referring to velocity and acceleration, which occur in a common tremor motion. A fixture was attached to the 6-DOF robot to mount the cup holder device. This way the stimulation of the device by use of different profiles of acceleration and different amplitudes was possible. The experiments were analysed by use of a digital video and a Fourier transform of the acceleration that could be measured. The experimental setup is shown in Fig. 7. It shows the robot with the fixture attached to its tool changer. On the fixture the cup holder device was 
mounted. To prevent the liquid spilling out of the cup and being poured on the robot an additional panel was attached.

In [25] experiments were conducted, which showed the frequencies resulting from the tremor of Parkinson patients to be in between 3 und $7 \mathrm{~Hz}$. The amplitude of the tremor could mostly be found below $0.3 \mathrm{~g}$, occasionally rising up to $0.9 \mathrm{~g}$. By use of this information the experiments could be specified. For the stimulation of the system a sinus function was used. Its range of frequencies was defined 3 to $7 \mathrm{~Hz}$. In general, the frequencies were varied in steps of $0.5 \mathrm{~Hz}$. In the most relevant range from 4 to $5 \mathrm{~Hz}$ the variation was done in steps of $0.25 \mathrm{~Hz}$. The amplitude was varied between $0 \mathrm{~g}$ and $0.45 \mathrm{~g}$ in steps of $0.05 \mathrm{~g}$. Amplitudes bigger than $0.45 \mathrm{~g}$ could currently not be realized due to safety issues during the use of the robot.

Every single movement of the 110 experiments conducted during the analysis uses an individual acceleration profile for the robot. For this purpose the robot was controlled by means of a micro controller to explicitly define the acceleration profile. The movement was stated linearly along the $x$-axis of the cup holder's sys coordinate system.

During the experiments the liquid's surface in the cup was monitored for spillage. In Fig. 8 the results of the experiments with and without the compensation are compared. The experiments in which no spillage of the beverage could be monitored are visualized in the graph. It is clear to see that in case of using the compensation method the amplitude and with it the acceleration of the tremor movement could be larger than without it.

It can also be seen, that at higher tremor frequencies spillage of the liquid gets unlikely, even without the compensation switched on. This can be explained by the fluid's eigenfrequency. At the filling level of the test setup it was calculated to $1.67 \mathrm{~Hz}$. So all tremor frequencies applied have been above eigenfrequency. In this range of frequency the resonance curve declines. Moreover, the amplitude of the liquid's oscillation declines with the increase of the frequency, due to the industrial robot's maximum acceleration. These effects reduce spillage at higher frequencies.

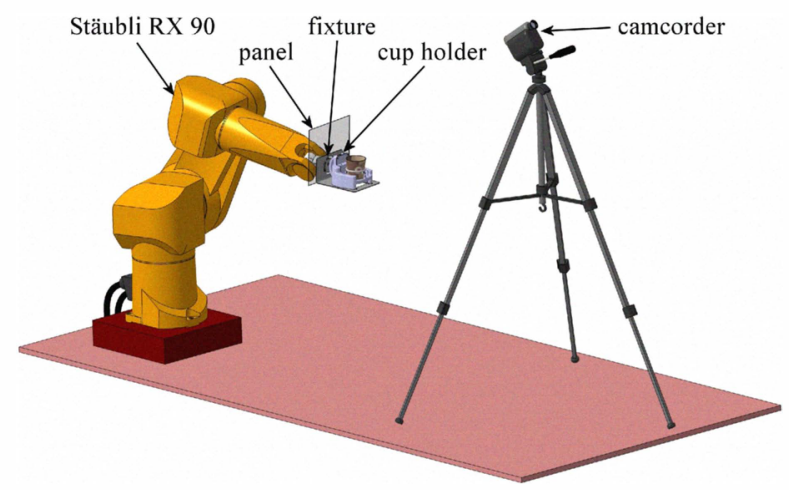

Figure 7. The testing environment: an industrial robot (Stäubli RX 90) which moves the cup holder, fastened by means of a fixture. The robot is shielded by a panel and a camcorder is used to record the experiments.

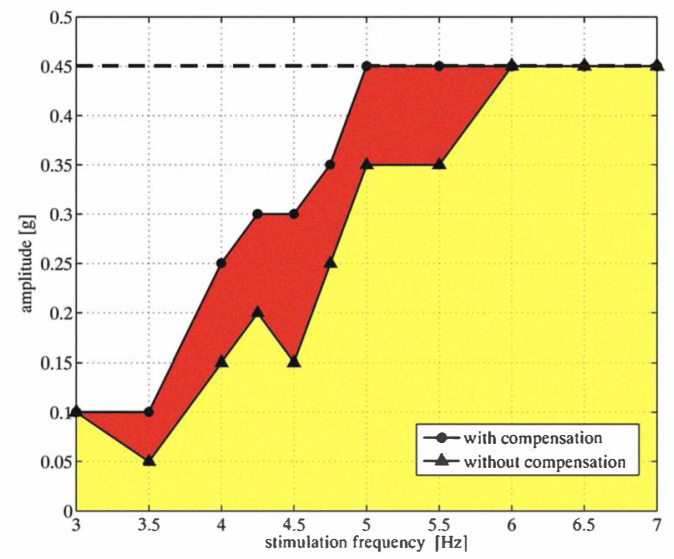

Figure 8. Illustration of the experiment's results: The amplitudes (and therewith the velocity) of the tremor without spillage can be higher when using the tremor compensation method. Amplitudes above $0.45 \mathrm{~g}$ have not been realized due to safety issues whilst using the robot.

\section{CONCLUSION AND DISCUSSION}

The work at hand presents a mechatronic device which improves ingestion for patients suffering tremor disease. More precisely the mobile system supports the drinking process by balancing the beverage's movements and therewith reduces spillage. After the build-up of the system's hardware, experiments were conducted to validate the sensor calibration as well as the developed equations. A control strategy for the system was implemented including a virtual pendulum for modelling the fluid's movement. At last, experiments for a validation of the system's operability were conducted. For this purpose an industrial robot was used. It moved the cup holder reproducible and measurable at a definite frequency as well as amplitude. Looking at the results of the experiments one can see that the system helps to reduce spillage of the beverage contained within the cup.

Compared to a patient's tremor, the shaking produced with the industrial robot is standardized. So in a next step of the work the system has to be tested on people. That way its effect on spillage and the acceptance by the users have to be evaluated. The tests could for example be conducted in hospitals or retirement homes. For this purpose two methods are conceivable. On the one hand, the system could be used to record the tremor of a group of patients during the drinking process. The information gathered could then be used to realize a reproducible, application oriented control of the robot. During further experiments, spillage of the liquid could be determined with or without the compensation function. On the other hand, the system could directly be tested in the normal routine of the day. The verification would then be more difficult but closer to reality.

Previous to these experiments, the current system has to be further improved. Both, the size and the weight of the cup holder could be reduced to improve the system's handling. Both parameters are partly due to the multitude of functions which are as yet implemented for evaluation reasons. In an improved system some of the interfaces wouldn't be necessary any more. Additionally, the micro controller and further elec- 
tronic components could be integrated into one single printed board. The accumulators used in the current system could as well be exchanged to reduce weight as well as size.

Noise, currently occurring during the determination of the accelerations, limits the accuracy of the calculations. To get a higher accuracy, the rotary accelerations could be detected more precisely. For that reason, sensors of a different type, e. g. gyro sensors, could be used. Furthermore, the distances between the sensors have a big influence on the accuracy due to the used calculation method. Hence these distances could be increased in order to increase accuracy.

Taking a look at the mechanics of the system an optimization of the parallel manipulator could be carried out. For example, the lengths of single elements could be optimized to increase the workspace of the robot. Beyond, the current kinematics setup could be improved using elements of higher quality. On the electronics side different actuators providing both higher acceleration and accuracy could be used.

\section{REFERENCES}

[1] W. Birkmayer, "Zur Differentialdiagnose des Tremors - Physikalische Analysen mit einer High-Speed-Kamera", Deutsche Zeitschrift für Nervenheilkunde, vol. 183, pp. 322-330, 1962.

[2] P. Faust, „Zittern (Tremor). Psychiatrie Heute - Seelische Störungen erkennen, verstehen, verhindern, behandeln", Arbeitsgemeinschaft Psychosoziale Gesundheit, 2004.

[3] G. Ellrichmann, Vorkommen und Wertigkeit von Oberfrequenzen in der 24-Stunden-Elektromyographie und Accelerometrie bei Parkinson und Essentiellem Tremor, Inaugural-Dissertation zur Erlangung des Doktorgrades der Medizin einer Hohen Medizinischen Fakultät der Ruhr-Universität Bochum, 2007.

[4] A. Berning, Prophylaxen in der Pflegepraxis: Risiken sicher einschätzen - Pflegestandards kompetent anwenden, Elsevier, München, 2007.

[5] W. Beitz and K.-H. Grote (ed.), Dubbel-Taschenbuch für den Maschinenbau, 20. edition, Springer, 2001.

[6] G. W. Brown, "Equipement for use with hand held motion picture cameras", Patent No. US 4.017.168, 12.04.1977.

[7] H. N. Abramson, W. H. Chu, and D. D. Kana, "Some studies of nonlinear lateral sloshing in rigid containers," Journal of Applied Mechanics, Transactions of the ASME, vol. 33, no. 4, pp. 777-784, 1966.

[8] K. Terashima and G. Schmidt, "Motion control of a cart-based container considering suppression of liquid oscillations", Proceedings of the International Symposium on Industrial Electronics. IEEE/ISIE' 94, pp. 275-280, 1994

[9] K. Yano and K. Terashima, "Robust Liquid Container Transfer Control for Complete Sloshing Suppression", IEEE Trans. on Control Systems Technology, vol. 9, no. 3, pp. 483-493, 2001.

[10] K. Yano, S. Higashikawa, and K. Terashima, "Motion control of liquid container considering an inclined transfer path", IFAC J. Contr. Eng. Practice, vol. 10, no. 4, pp. 465-472, 2002
[11] R. Graf and R. Dillmann, "Active acceleration compensation using a Stewart-platform on a mobile robot", Proceedings of the 2nd Euromicro Workshop on Advanced Mobile Robots, EUROBOT '97, Brescia, Italy, pp. 59-64, 1997.

[12] R. Graf, R. Vierling, and R. Dillmann, "A flexible controller for a stewart platform", 2nd International Conference on Knowledge-Based Intellegent Electronic Systems - KES 98, vol. 2, pp. 52-59, 1998.

[13] M. W. Decker, A. X. Dang, and I. Ebert-Uphoff, "Motion Planning for Active Acceleration Compensation", Proceedings of the 2001 IEEE International Conference on Robotics \& Automation, Seoul, Korea, vol. 2, pp. 1257-1264, 2001.

[14] A. X. Dang and I. Ebert-Uphoff, "Active Acceleration Compensation for Transport Vehicles Carrying Delicate Objects", IEEE Transactions on Robotics, vol. 20, no. 5, pp. 830-839, 2004.

[15] D. Lanska, "19th-century American contributions to the recording of tremors", Movement Disorders, vol. 15, no. 4, pp. 720-729, 2001.

[16] J. Matsumoto, D. Dodick, L. Stevens, R. Newman, P. Caskey, and W Fjerstad, "Three-dimensional measurement of essential tremor", Movenment Disorders, vol. 14, no. 2, pp. 288-294, 1999.

[17] M. H. Asyali and T. Dalbasti, "An Image Analysis Based Method For The Quantification Of Tremor", Journal of Yasar University, vol. 2, no. 7, pp. 631-640, 2007.

[18] C. F. Huang, S. S. Huang, Yang Meng-Hsiang, M. S. Young, K. Y. Lee, and R. C. Chen, "A new ultrasonic measurement system for tremor", IEEE EMBS Asian-Pacific Conference on Biomedical Engineering, pp. 260-261, 2003

[19] A. Legros, N. Diakonova, L. Cif, S. Hemm, N. Vayssière, P. Coubes, and A. Beuter, "Accelerometric measurement of involuntary movements during pallidal deep brain stimulation of patients with generalized dystonia", Brain Research Bulletin 64, pp. 363-369, 2004.

[20] H. Dejnabadi, B. M. Jolles, E. Casanova, and P. Fua, "Estimation and Visualization of Sagittal Kinematics of Lower Limbs Orientation Using Body-Fixed Sensors", IEEE Transactions on Biomedical Engineering, vol. 53, no. 7, pp. 1385-1393, 2006.

[21] R. Taylor, P. Jensen, L. Whitcomb, A. Barnes, R. Kumar, D Stoianovici, P. Gupta, Z. Wang, E. de Juan Jr., and L. Kavoussi, "A steady-hand robotic system for microsurgical augementation," Proceedings of the 1999 International Conference on Robot Research, vol. 18. pp. 1201-1210, 1999.

[22] B. Mitchell, J. Koo, I. Iordachita, P. Kazanzides, A. Kapoor, J. Handa, G. Hager, and R. Taylor, "Development and Application of a New Steady-Hand Manipulator for Retinal Surgery," Proceedings of the 2007 IEEE International Conference on Robotics and Automation, Roma, Italy, pp. 623-629, 2007.

[23] W. T. Latt, U-X. Tan, C. Y. Shee, and W. T. Ang, "A Compact Handheld Active Physiological Tremor Compensation Instrument", Proceedings of the 2009 IEEE/ASME International Conference on Advanced Intelligent Mechatronics, Singapore, pp. 711-716, 2009.

[24] C. Peters, A. Buhmann, and Y. Manoli, "Method and device for determining the relative position, speed and/or acceleration of a body", Patent No. WO 2006/128592 A1, 07.12.2006.

[25] F. Foerster, T. Thielgen, G. Fuchs, A. Hornig, and J. Fahrenberg, "24stündige Tremor-Untersuchungen an Parkinson-Patienten “, Forschungsberichte des Psychologischen Instituts der Albert-LudwigsUniversität Freiburg i. Br., Nr. 151, 2001. 